\title{
Integral observations of polarization: from the Crab pulsar to Cygnus X-1
}

\author{
P. Laurent ${ }^{* a}$, D. Götz ${ }^{b}$, C. Gouiffès ${ }^{b}$, V. Grinberg ${ }^{c}$, P. Moran ${ }^{d}$, J. Rodriguez $^{b}$, J. Wilms $^{c}$ \\ ${ }^{a} A P C$, Astroparticule et Cosmologie, Université Paris Diderot, CNRS/IN2P3, CEA/Irfu, \\ Observatoire de Paris, Sorbonne Paris-Cité, 10, rue A. Domon et L. Duquet, 75205 Cedex 13, \\ France \\ ${ }^{b}$ AIM, CEA/IRFU, CNRS/INSU, Université Paris Diderot, CEA DSM/IRFU/SAp, 91191 Gif sur \\ Yvette, France \\ ${ }^{c}$ Dr. Karl-Remeis-Sternwarte Astronomisches Institut der Universität Erlangen-Nürnberg, \\ Sternwartstr. 7, 96049 Bamberg, Germany \\ ${ }^{d}$ Centre for Astronomy, National University of Ireland, Galway, Ireland \\ E-mail: plaurentecea.fr
}

\begin{abstract}
In complement to spectro-imaging observations, $\gamma$-ray polarimetry provides a unique insight into the geometry and magnetic configuration of compact $\gamma$-ray sources, such as neutron stars or black holes. Due to the unprecedented spectral and timing capabilities of Integral, and thanks to its coded mask imaging technics, which efficiently suppresses most of the background contribution, we have measured linearly polarized emission from the brightest cosmic high energy sources with the two telescopes IBIS and SPI. We were able to measure for the first time, at energies above $200 \mathrm{keV}$, a clear polarization signal from different types of $\gamma$-ray sources such as the Crab pulsar and nebula, the black hole candidate Cygnus X-1, and Gamma-Ray Bursts. These observations have enabled us to put strong constraints on the physical process at work in these sources, and the achieved sensitivity opens a new window for polarimetric studies in the soft $\gamma$-ray regime.
\end{abstract}

"An INTEGRAL view of the high-energy sky (the first 10 years)" 9th INTEGRAL Workshop and celebration of the 10th anniversary of the launch,

October 15-19, 2012

Bibliotheque Nationale de France, Paris, France

\footnotetext{
* Speaker.
} 


\section{Introduction}

Whereas difficult, $\gamma$-ray polarimetry has been possible with Compton telescopes since the 1970s. Photons that are Compton scattered between two detectors follow an azimuthal distribution around the source direction that allows to quantify the degree and direction of linear polarization because these photons are preferentially scattered in a plane at right angle to their incident electric vector. This measure was unsuccessful up to recently because of intrinsic asymmetries in the detector response and of non-uniformities in the large background signals. These defaults induce pseudo polarimetric signals, even from an unpolarized source, that limit the sensitivity to any detection. Thanks to their imaging capacities, however, the IBIS [1] and SPI [2] telescopes on board the Integral satellite ${ }^{1}$ are well suited for polarimetry studies, between $200 \mathrm{keV}$ and $5 \mathrm{MeV}$. In this paper, we will describe the polarimetry methods developed, and present evidence for the detection of polarization from the Crab nebula, Gamma-Ray Bursts, and Cygnus X-1 at energies above 200 $\mathrm{keV}$.

\section{Polarimetry with the Integral/IBIS Compton mode}

Photons entering the INTEGRAL/IBIS telescope may be Compton scattered in the first detector plane, ISGRI [3], at a polar angle $\theta$ from their incident direction and at an azimuth $\psi$ from their incident electric vector. They are then absorbed in the second detector, PiCsIT [4]. The azimuthal profile $N(\psi)$, in Compton counts recorded per azimuth bin, follows:

$$
N(\psi)=S\left[1+a_{0} \cos \left(2 \psi-2 \psi_{0}\right)\right]
$$

for a source polarized at an angle $\mathrm{PA}=\psi_{0}-\pi / 2+n \pi$ and with a polarization fraction $\mathrm{PF}=$ $a_{0} / a_{100}$. The $a_{100}$ amplitude is expected for a $100 \%$ polarized source. Unfortunately, the IBIS and SPI polarimetric capacities have not been calibrated on ground, due to the tight planning of the INTEGRAL project before launch. For IBIS, we have then evaluated $a_{100}$ to be $0.30 \pm 0.02$ for a Crab-like $E^{-2.2}$ spectrum between 200 and $800 \mathrm{keV}$, using GEANT3 Monte-Carlo simulations with the GLEPS package ${ }^{2}$ for polarization.

Events recorded in ISGRI and PiCsIT within the same time window of $3.8 \mu \mathrm{s}$ are tagged as 'Compton' events, but do not all result from Compton scattering. Chance coincidences can occur between ISGRI and PiCsIT events independently coming from the source, the sky, or the instrumental background. These coincidences are generally called spurious events. Most of the 'Compton'-tagged events are due to background events that will be removed by the shadowgram deconvolution; $5 \%$ are due to a small fraction of spurious coincidences that must be removed with high accuracy because they induce a false source detection in the sky image; $2 \%$ come from true Compton events from the source.

The procedure we used to substract the spurious events contribution and measure the polarization is described in Forot et al. [5]. Confidence intervals on $a_{0}$ and $\psi_{0}$ are not given by the $N(\psi)$

\footnotetext{
${ }^{1}$ Integral is an ESA project with instruments and science data center funded by ESA member states with the participation of Russia and USA. ISGRI has been realized and maintained in flight by CEA/Irfu with the support of CNES.

${ }^{2}$ GLEPS is a package for handling polarization in Geant 3 developed by Dr. Mark McConnell at University of New Hampshire, USA
} 
fit to the data since the variables are not independent. They have been derived from the probability density distribution of measuring a and $\psi$ from $N_{p t}$ independent data points in $N(\psi)$ over a $\pi$ period, based on Gaussian distributions for the orthogonal Stokes components [6]:

$$
d P(a, \psi)=\frac{N_{p t} S^{2}}{\pi \sigma_{S}^{2}} \exp \left[-\frac{N_{p t} S^{2}}{2 \sigma_{S}^{2}}\left[a^{2}+a_{0}^{2}-2 a a_{0} \cos \left(2 \psi-2 \psi_{0}\right)\right]\right] a d a d \psi
$$

$\sigma_{S}$ notes the error on the profile mean S. The errors on each a or $\psi$ dimension are obtained by integrating $\mathrm{dP}(\mathrm{a}, \psi)$ over the other dimension.

\section{Polarimetry with the SPI telescope}

The SPI telescope onboard Integral is able to measure $\gamma$-ray sources polarization through an analysis of its "multiple events". Indeed, these events are generated when a photon makes several energy deposits in two or more SPI germanium detectors, and are mostly due to Compton scattering in different detectors. They can thus be used to measure polarimetry, on the same basis as what is done for the IBIS/Compton mode. However, due to the SPI imaging complexity, it is merely impossible to measure this polarization directly, and one has to rely on heavy Monte-Carlo simulations to look for a polarized signal in the data. This search is done by computing, for each pointing and a given source spectrum, overall SPI simulations for 100\% polarized sources with 18 given position angles spanning from $0^{\circ}$ to $180^{\circ}$ and also using an unpolarized source. Simulation results for a given polarization fraction and angle are then given by [7]:

$$
G 4(a, \psi)=a G 4(\psi)+(1 .-a) G 4(0)
$$

where $\mathrm{G} 4(\psi)$ and $\mathrm{G} 4(0)$ are the results of GEANT-4 simulations for beams with $100 \%$ polarization, polarization angle $\psi$, and for unpolarized beams, respectively. The simulated count rates for both cases are then compared with a $\chi^{2}$ statistics with the observed distribution. Polarized angle and fraction are thus determined by the simulation giving the lowest $\chi^{2}$. This method was first described by Dean et al. [8], and then improved in the recent Jourdain et al. paper on Cygnus X-1 [7].

\section{Crab observations}

The first studies of the Crab polarization at hard X-ray energies by Integral have been published by Dean et al. [8] and Forot el al. [9]. In this last paper, we use Integral observations of the Crab nebula between 2003 and 2007 for a total of $1.2 \mathrm{Ms}$. The pulsed lightcurve in the $200-800 \mathrm{keV}$ band has been constructed with the Jodrell Bank ephemerides of the pulsar. We have considered four phase intervals for polarimetry studies : the two main peaks, the off-pulse interval and the bridge interval, dominated by the nebular emission. The interval boundaries were taken from [10] and were not adapted to enhance a possible signal. Table 1 and Fig. 1 show the results and illustrate the contrast between the modulation obtained for the nebular emission versus the flat profile of the pulsed emission.

There is indeed no significant indication of polarization in the pulsed peaks. The chance probability of a random fluctuation reaches $33.5 \%$ and the signal shows no modulation at the $95 \%$ confidence level over all angles. This behaviour is consistent with the radio and optical data where 

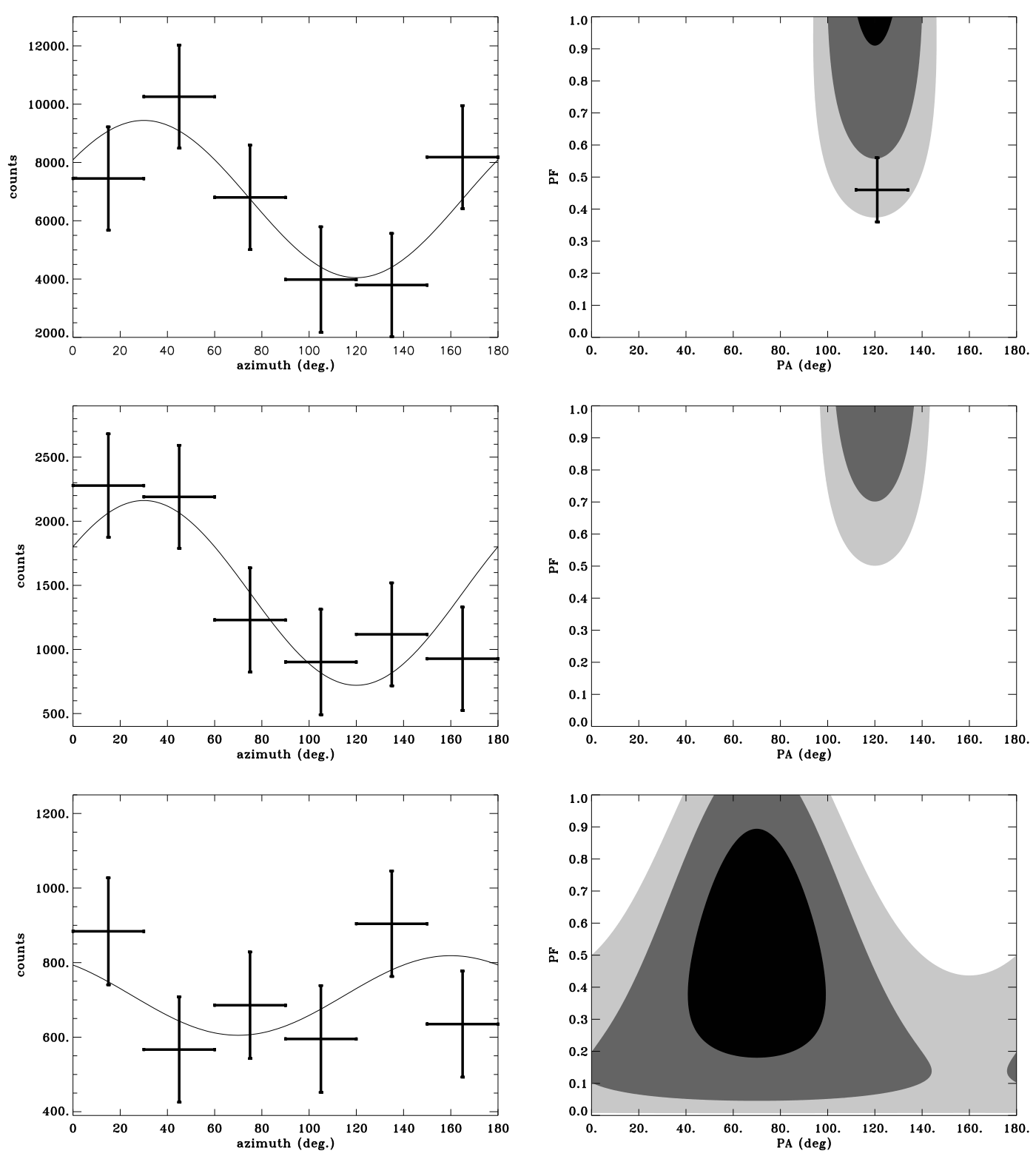

Figure 1: Azimuthal profile, modulation angle, PA, and fraction, PF, measured in the IBIS Compton mode for the Crab data between 200 and $800 \mathrm{keV}$, in the off-pulse (top), off-pulse and bridge (middle), and twopeak (bottom) phase intervals. The error bars for the profile are at one sigma. The 68\%, 95\%, and $99 \%$ confidence regions are shaded from dark to light gray. The SPI result [8] is indicated in the top figure by a cross. 
Table 1: Polarization angle and fraction with respect to the Crab pulsar phase $\phi$

\begin{tabular}{cccc}
\hline \hline phase interval & polarization angle & polarization fraction & chance probability \\
\hline $\mathrm{P}_{1}$ and $\mathrm{P}_{2}(\mathrm{IBIS})$ & $70^{\circ} \pm 20^{\circ}$ & $0.42_{-0.16}^{+0.30}$ & $33.5 \%$ \\
$\mathrm{OP}(\mathrm{IBIS})$ & $120.6^{\circ} \pm 8.5^{\circ}$ & $>0.72^{a}$ & $0.26 \%$ \\
$\mathrm{OP}(\mathrm{SPI})$ & $123^{\circ} \pm 11^{\circ}$ & $0.46 \pm 0.1$ & \\
OP and $\mathrm{B}(\mathrm{IBIS})$ & $122.0^{\circ} \pm 7.7^{\circ}$ & $>0.88^{a}$ & $0.10 \%$ \\
all $(\mathrm{IBIS}, 2003-2007)$ & $100^{\circ} \pm 11^{\circ}$ & $0.47_{-0.13}^{+0.19}$ & $2.8 \%$ \\
all(IBIS, $2007-2012)$ & $85^{\circ} \pm 10^{\circ}$ & $0.58 \pm 0.07$ & \\
\hline \hline
\end{tabular}

a : The lower limits for the polarization fraction are given at the $95 \%$ confidence level.

PF drops below $10 \%$ as the angle largely flips within each peak [11]. Conversely, the chance is low that the modulation seen in the off-pulse emission above $200 \mathrm{keV}$ be of random origin. The $\mathrm{P}(\mathrm{a}, \psi)$ probability density yields a probability of $2.610^{-3}$ that a random fluctuation produces an amplitude $a_{0}$ larger than the recorded one. Adding the bridge and off-pulse data strengthens the signal and gives a chance probability of $10^{-3}$ that an unpolarized source produce this modulation (see table 1). The off-pulse-and-bridge emission is polarized at an angle of $122.0^{\circ} \pm 7.7^{\circ}$ which is fully consistent with the north-to-east angle $\psi=124^{\circ} \pm 0.1^{\circ}$ of the pulsar rotation axis projected on the skyplane.

We have pursued this study of the Crab pulsar and nebula polarization using the 2007 to 2012 Integral/IBIS data. The results we found for the whole pulsar period are shown on the last row of Table 1 and are consistent with those obtained in the Forot et al. paper (see P. Moran et al., [12] for details).

\section{Observation of variable polarization from Gamma Ray Bursts}

The IBIS and SPI telescopes on board the INTEGRAL satellite have also been used to measure the polarization of the prompt $\gamma$-ray emission of long and bright gamma-ray bursts (GRB) in the $200-800 \mathrm{keV}$ energy band. Two bright GRBs have been studied so far by Integral/IBIS: GRB 041219A and GRB 061122. For GRB 041219A, characterized by a two peaked light curve, Götz et al. [13] found a variable degree of polarization ranging from less than $4 \%$ over the first peak to $43 \pm 25 \%$ for the whole second peak. Time resolved analysis of both peaks indicates a high degree of polarization, and the null average polarization in the first peak can be explained by the rapid variations observed in the polarization angle and degree. The azimuthal distributions of the GRB flux for the different time intervals are reported in figure 2.

These results are consistent with the SPI analysis which reported a high polarization level (68\% with $\psi=70^{\circ} \pm 12^{\circ}$ [14]) observed during the brightest part of GRB041219A, corresponding to the P8 time interval in figure 2 , when IBIS measured a polarization angle $\psi=88^{\circ} \pm 12^{\circ}$. Finally, using a recent determination [15] of the GRB distance, and measuring the polarization properties of the emitted photons in two adjacent energy range, Laurent et al. [16] were able to increase by 4 orders of magnitude the existing constraint on Lorentz invariance violations (LIV), arising from 


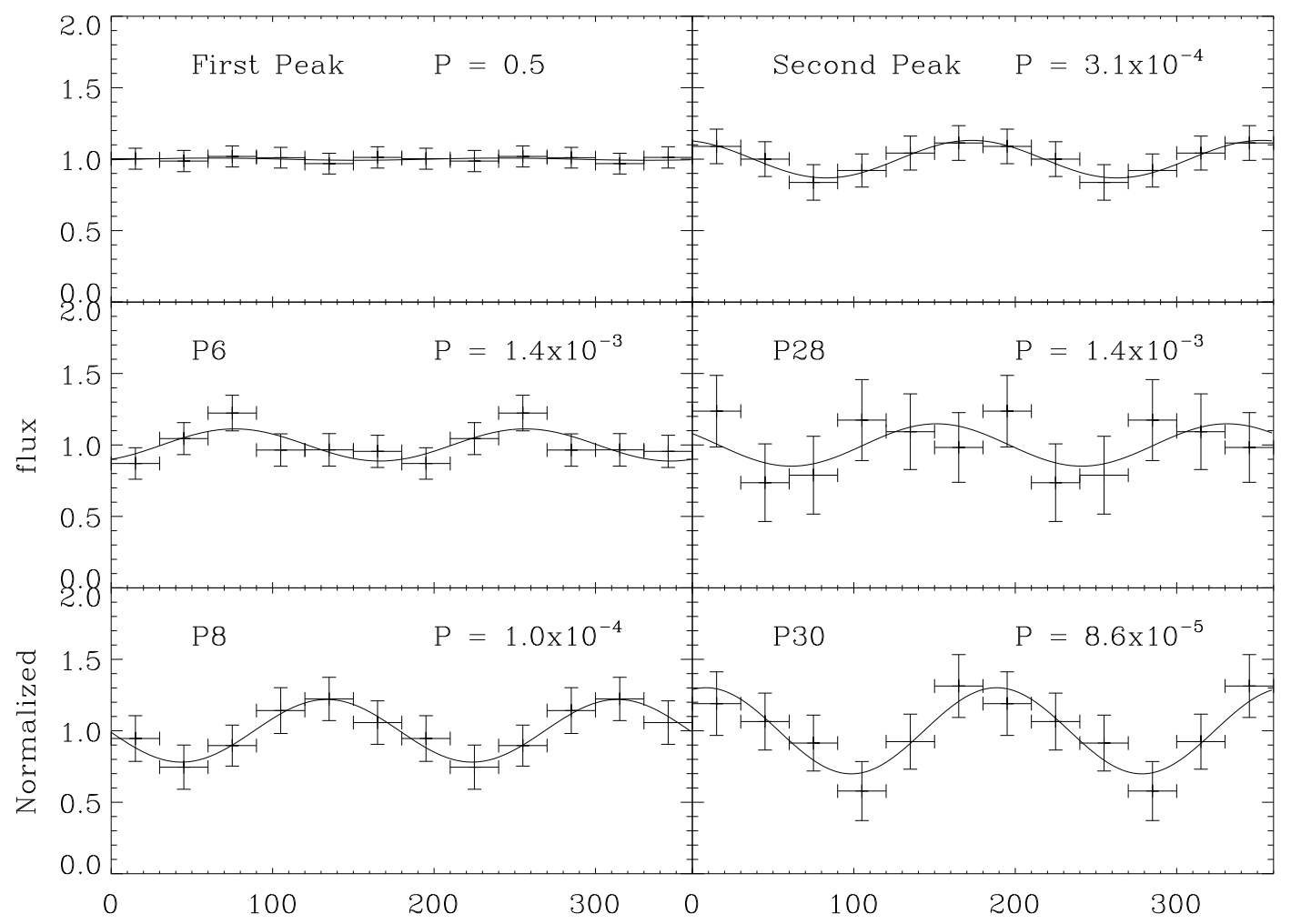

Figure 2: Polarigrams of the different GRB 041219A time intervals that have been analyzed. For comparison purposes, the curves have been normalized to their average flux level. For a definition of the time intervals see [13].

vacuum birefringence. The study of GRB 041219A and GRB 061122 polarization with Integral is described in more details in [17].

\section{Observation of the Cygnus $\mathrm{X}-1$ polarized high energy component}

Cygnus $\mathrm{X}-1$ is probably the best known black hole $(\mathrm{BH}) \mathrm{X}$-ray binary in our Galaxy. It is located around $1.9 \mathrm{kpc}$ away from the Earth, and forms a binary system together with a high mass blue $\mathrm{O}$ star. It radiates mainly in the X-ray and soft gamma-ray domain and its X-ray luminosity is thought to come from accretion of matter from the companion onto the $\mathrm{BH}$ through the formation of an accretion disk. Finally, a compact radio jet is ejected from the vicinity of the BH, with a kinetic power similar to the source's bolometric X-ray luminosity (see [18] and references therein). The spectral measurements of the INTEGRAL/IBIS/Compton observations of Cygnus X-1 are shown in Figure 3 [18], where the X-ray/gamma-ray spectrum as obtained with the standard Integral/IBIS pipeline is presented. Two high energy components are clearly seen, a cutoff power law component between 20 and $400 \mathrm{keV}$, reminiscent of a Compton-scattering induced spectrum, and a power law 
spectrum at higher energies of up to $2 \mathrm{MeV}$. These two components are signatures of two different high energy emission processes in the source, whose locations have not yet been constrained.

Thanks to polarization measurements, we are now able to better understand this gamma-ray emission geometry. Indeed, the signal measured in the first energy band for which a polarimetry study could be performed (250-400 keV), appeared to be consistent with a non-polarized signal with an upper limit of $20 \%$ for the polarization fraction PF (shown in Figure 3). This result agrees with what is expected from a zone where Compton multiple scattering dominates. In contrast to the low polarization in the 250-400 keV band, the signal from the 400-2000 keV band in which the hard tail dominates is highly polarized ( $\mathrm{PF}=67 \pm 30 \%$, see Figure 3 ). This result is no longer consistent with domination of Compton scattering, and such a high polarization fraction is more likely the signature of synchrotron emission, e.g., from the jet already observed in the radio band.

A similar spectral dependence of the polarization signal from Cygnus X-1 has been recently observed by the SPI telescope [7]. IBIS and SPI are thus now consistently measuring a highly polarized signal above $400 \mathrm{keV}$ at a polarization angle $\psi=40^{\circ} \pm 8^{\circ}$, that is $60^{\circ}$ away from the compact radio jet position $\left(-21^{\circ}:-24^{\circ}[19]\right)$.

Following these results, we aim to study in the near future with Integral the variation of the polarimetric signal with the different Cygnus X-1 spectral states, using the spectral state determination described in [20], and to use Integral long term observations to measure possibly polarized signals from other bright Black Hole candidates, such as GX 339-4, GRS 1915+105 or Sw J174526.

\section{Conclusions}

The measurement of polarization in hard- $X$ rays/soft $-\gamma$ rays is a powerful tool to investigate the emission mechanisms and geometry of high-energy astrophysical sources. We have shown that fundamental physics questions can also be addressed. By these pioneering observations, Integral is a pathfinder and we hope that next generation dedicated polarimeters (e.g. POLAR [21], AstroH/SGD [22], etc.) will complement the Integral discoveries.

\section{References}

[1] Ubertini, P., et al. 2003, A\&A, 411, L131

[2] Vedrenne G., et al. 2003, A\&A,411,L63

[3] Lebrun, F., et al. 2003, A\&A, 411, L141

[4] Labanti, C., et al. 2003, A\&A, 411, L149

[5] Forot, M., Laurent P., Lebrun F., \& Limousin O., 2007, ApJ, 668, 1259

[6] Weisskopf, M. C., 2006, Proc. of Neutron Stars and Pulsars, Bad Honnef, Germany

[7] Jourdain E., et al. 2012, ApJ, 761, 27

[8] Dean, A.J., et al. 2008, Science, 321, 1183

[9] Forot M., Laurent P., Gouiffes C., Grenier I., \& Lebrun F., 2008 ApJ, 688, L29 


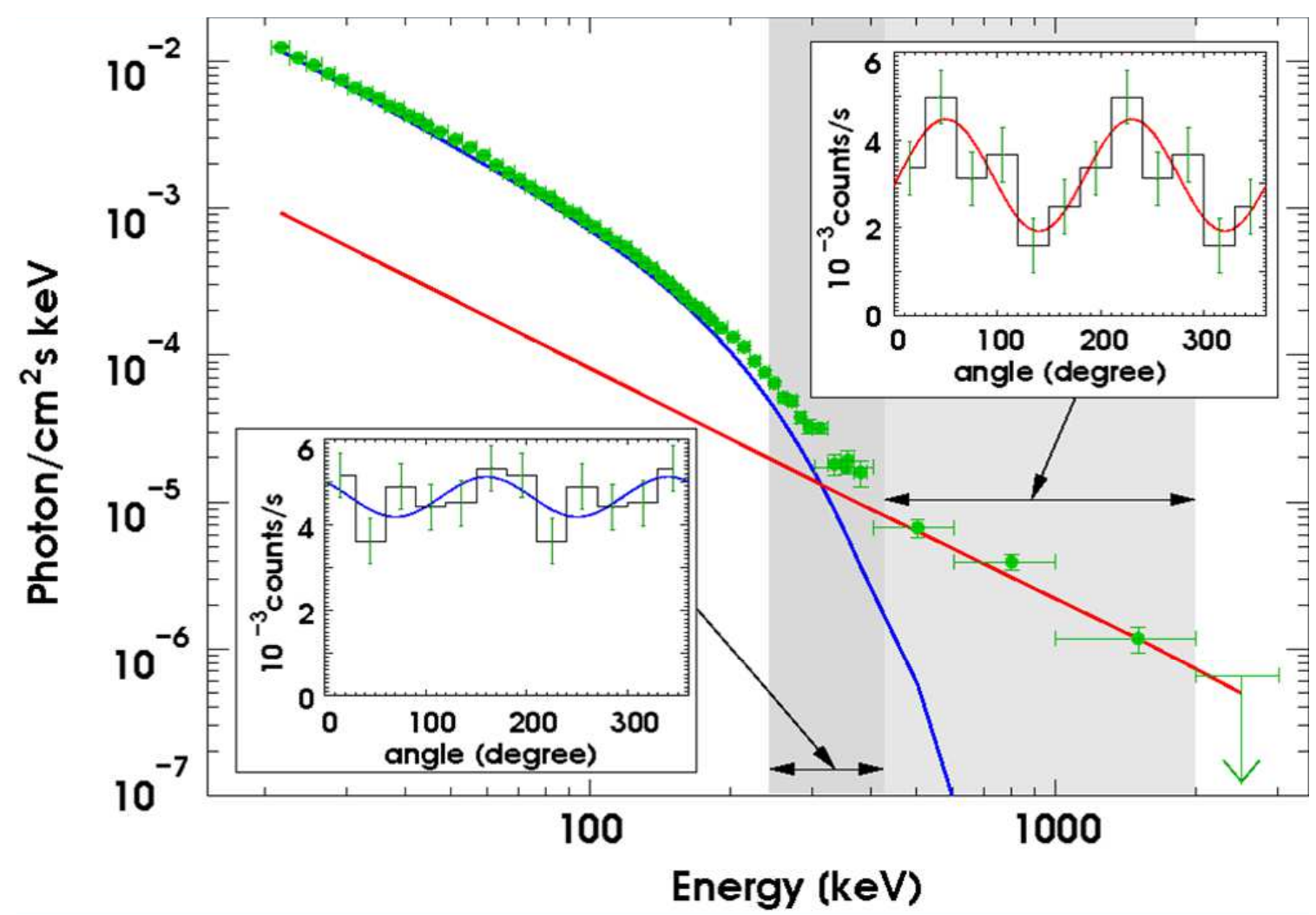

Figure 3: Spectra and polarigrams observed by the Integral/IBIS telescope from Cygnus X-1.

[10] Kuiper, L., Hermsen, W., Cusumano, G., Diehl, R., Schonfelder, V., Strong, A., Bennett, K., \& McConnell, M. L. 2001, A\&A, 378, 918

[11] Slowikowska, A., Kanbach, G., Kramer, M., \& Stefanescu, A. 2008 Proc. of high time resolution astrophysics: The Universe at Sub-Second Timescales. AIP Conference Proceedings, 984, 51

[12] Moran, P. et al., 2012, these proceeding

[13] Gotz D., Laurent P., Lebrun F., Daigne F.,Bosnjak Z., 2009, ApJ, 695, L208

[14] Mc Glynn S. et al., 2007, A\&A, 466, 895

[15] Gotz D., Covino S., Hascoet R., Fernandez-Soto A.,, Daigne F., Moskovitch R., Esposito P., 2011, MNRAS 413, 2173

[16] Laurent P., Gotz D., Binétruy P., Covino S., Fernandez-Soto A., 2011, Phys.1 Rev. D, 83, 12, 121301

[17] Gotz D., et al., 2012, these proceedings

[18] Laurent P., Rodriguez J., Wilms J., Cadolle Bel M., Pottschmidt K., Grinberg V., 2011, Science, 332 , 6028,438

[19] Stirling A.M., et al., 2001, MNRAS, 327, 1273

[20] Grindberg, V. et al., 2012, these proceedings

[21] Produit N., et al. 2005, NIMPA, 550, 616

[22] Takahashi T., et al., 2012, SPIE conf. proc., 8443, $1 \mathrm{Z}$ 